\title{
Analysis on transient behavior of cable-towed acoustic array
}

\author{
Jing Biao ${ }^{\mathrm{a}}$, Zhuke Qiang ${ }^{\mathrm{b}}$, Zhang Yang ${ }^{\mathrm{c}}$ \\ Department of Marine and Transportation, Ningbo University, 315211,China \\ aemail:15728046516@163.com, bemail: zhukeqiang@nbu.edu.cn, email:zhangyang182@yeah.net
}

Keywords: Cable-towed acoustic array; cable dynamics; transient behavior; close-loop radius

\begin{abstract}
This paper reports on dynamic characteristics of a cable-towed acoustic array system during ship's loop turning maneuvers with a lumped parameter model. The transient behavior of the towed sonar array given rise to the tow-ship's loop maneuvers with different towing speeds, tuning radii, and current are intensively examined via numerical simulation, the corresponding results provide an insight into the tactical aspects of cable-towed array system’s applications.
\end{abstract}

\section{Introduction}

As underwater systems that towed behind a submarine or a surface vessel, cable-towed acoustic arrays have been widely used in ocean oil prospecting and anti-submarine warfare. The detection and analysis of distant or silent targets is the main task of towed sonar array systems. In order to overcome the shortcomings of port/starboard ambiguity and determine the location of candidate underwater target, the tow ship has to take some certain tuning maneuvers during the detecting of acoustic source. Therefore, it is of great importance to accurately predict the dynamic behavior of the cable-towed sonar array during tow vessel in turning maneuvers.

Many articles have concentrated on model development or algorithm optimization ${ }^{[1-4]}$, few of them have given an insight into practical problems of cable-towed systems. Most of the present knowledge about the configuration of an undersea tow cable during ship turning can be attributed to the steady-state analyses of $\mathrm{Choo}^{[5]}$, Chapman $^{[6]}$ and Grosenbaugh ${ }^{[7]}$. Compared to Choo, the other two gived more comprehensive studies on towed body system. Chapman defined a critical radius, when the ship conducts $360^{\circ}$ circular turning at a given towing speed, the cable or towed body forms an equilibrium shape that is almost equal to plane shape of the ship trajectory. In this case, the turning radius and velocity of the vehicle are nearly equal to that of the ship. Grosenbaugh extended Chapman's work by performing a detailed analysis of the transient behavior of the towed system from a dynamic standpoint and developing an alternate definition for critical turning radius. Kishore ${ }^{[8]}$ performed a fairly comprehensive study on the dynamic behavior of the towed sonar array during ship in $360^{\circ}$ loop maneuvers and focused on the close-loop response of the cable-towed acoustic array with the sensitivity analysis of the ratio of tow cable length to ship-turning radius and tow-speed. He pointed that the cable-towed array started forming a close-loop when the ship-turning radius decreased to a specific value, we define it as close-loop radius in our present study since a close-loop is formulated then.

The motivation of our work is to extend the precious works by performing a detailed analysis of the cable-towed acoustic array's transient behavior that occurs in starting from a steady straight-towing configuration to a steady-state turning configuration. We will focus on the effect of towing speed, turning radius, and current on the dynamic response on towed sonar array during ship in loop turning maneuvers, which may help to plan ship towing strategy to optimize stable listening conditions for the acoustic array during actual underwater surveillance. It is to be noted that the towing platform considered in the following cases is a surface ship.

\section{Numerical methods}

A lumped parameter cable model, which was proposed by Yang et al [9], is adopted in our study. The governing equations system on which the mathematical model is based include the effects of 
bending stiffness and geometric nonlinearities for modeling of low-tension model.

The case parameters used in all our simulations are chosen from the work published by Gobat and Grosenbaugh [4]. The schematic of cable-towed array is shown in Fig. 1. The detailed physical properties of each segment of the towed array are listed in Tab. 1, and the symbols in it are consistent with those in Yang et al [9].

Tab.1. Parameters of the cable-towed

\begin{tabular}{cccc}
\hline & Tow cable & array & drogue \\
\hline $\mathrm{EA} / \mathrm{N}$ & $1 \times 10^{8}$ & $1 \times 10^{8}$ & $5 \times 10^{6}$ \\
$\mathrm{EI} / \mathrm{Nm}^{2}$ & 1000 & 1000 & 0.01 \\
$\mathrm{~m} / \mathrm{kg} \cdot \mathrm{m}^{-1}$ & 2.37 & 5.07 & 0.58 \\
$\mathrm{G} / \mathrm{N} \cdot \mathrm{m}^{-1}$ & 2.33 & 0 & 0.57 \\
$\mathrm{~d} / \mathrm{m}$ & 0.041 & 0.079 & 0.025 \\
$C_{\tau}$ & 2.0 & 1.8 & 1.8 \\
$C_{n}$ & 0.015 & 0.009 & 0.022 \\
\hline
\end{tabular}

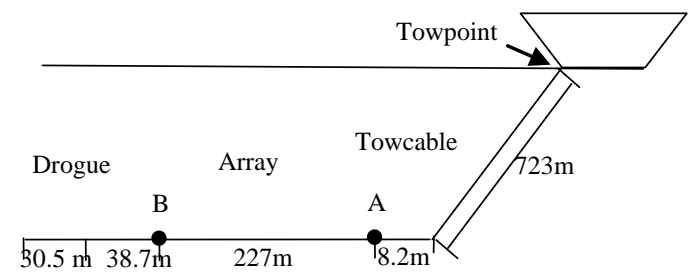

Fig.1. Schematic of cable-towed array

\section{Results and Discussions}

\section{Different towing speed}

To investigate the effect of change in the towing speed on the dynamics of the towed array during ship in circular maneuver, three cases have been simulated with different towing speed $(V=$ $18,6$, and $3 \mathrm{kn})$. The turning radius is $400 \mathrm{~m}$.
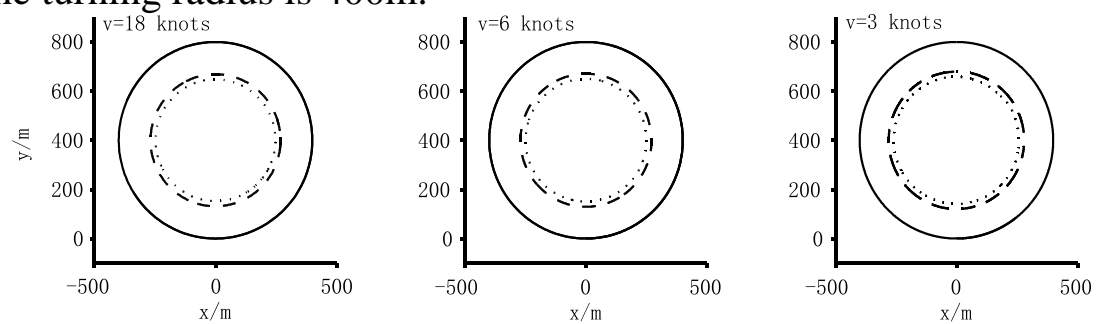

Fig.2. Plan view of loop maneuvers for $R=400 \mathrm{~m}, V=18$, 6, and $3 \mathrm{kn}$.

— represents tow-point, - - - represents point A, : represents point B
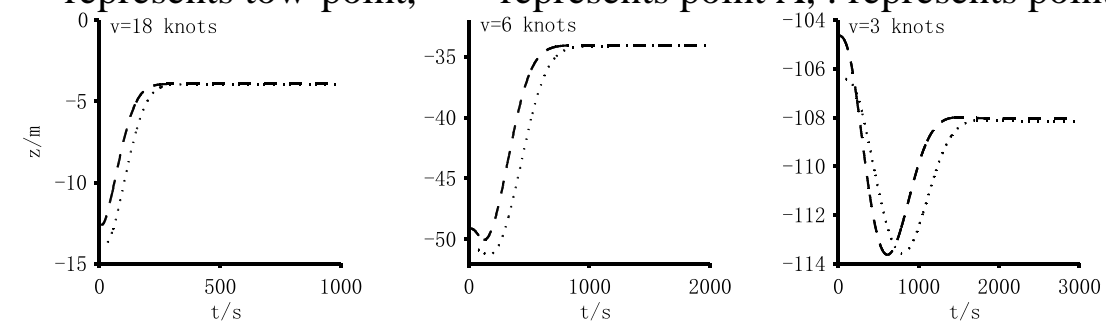

Fig.3. Time history of depth during loop maneuvers for $R=400 \mathrm{~m}, V=18$, 6, and $3 \mathrm{kn}$.

- - - represents point $A$, : represents point $B$

Few differentiations are found in the plan view of loop maneuver with the change of towing speed. The turning radius of point A and B is unanimous under different towing speed (Fig. 2).

As the tow speed of the loop maneuver with a constant loop radius is reduced, the depths of point $\mathrm{A}$ and $\mathrm{B}$ on the cable-array have dramatic changes, as can be seen from Fig. 3. For case with $\mathrm{V}=18$ $\mathrm{kn}$, the depths of point $\mathrm{A}$ and $\mathrm{B}$ decrease directly and achieve their new balanced-positions soon. For case with $\mathrm{V}=6 \mathrm{kn}$, the depths of point $\mathrm{A}$ and $\mathrm{B}$ obtain their steady-state after a temporary slight 
drop and a significant fast rise. For case with $\mathrm{V}=3 \mathrm{kn}$, the final depths for point $\mathrm{A}$ and $\mathrm{B}$ in Fig. 3 are lower than the initial ones, which differs from the former two samples. According to the plots of the lowest speed, we can find that the depth of point A is lower than that of point B in a certain period before the towed array arrives in final steady-state. On the contrary, there is no such situation in the plots of the other two relatively higher speed $(\mathrm{V}=18,6 \mathrm{kn})$.

\section{Different turning radii}

A comparison is made as the follow pictures to investigate the effect of the turning radii on the dynamics of the towed array during ship in circular maneuver. Given a constant tow speed ( $\mathrm{V}=6$ $\mathrm{kn})$, three cases with different turning radius $(\mathrm{R}=800,400$, and $300 \mathrm{~m}$ ) have been simulated, and the results are given as:
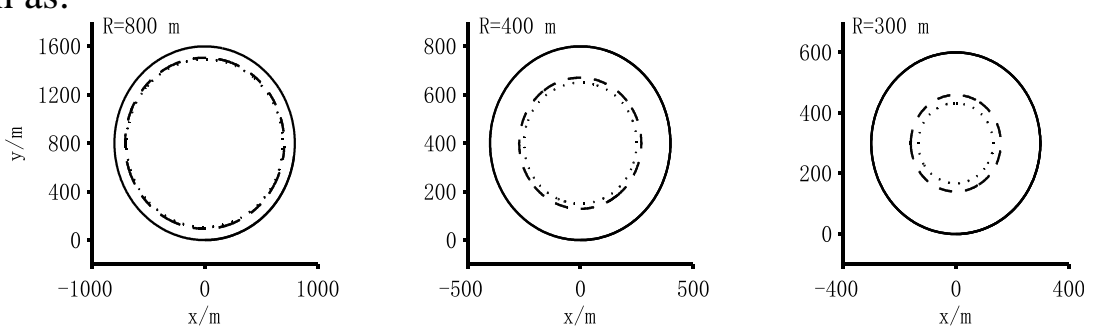

Fig.4. Plan view of loop maneuvers for $V=6 \mathrm{kn}, R=800$, 400, and $300 \mathrm{~m}$.

- represents tow-point, - - - represents point A, : represents point B
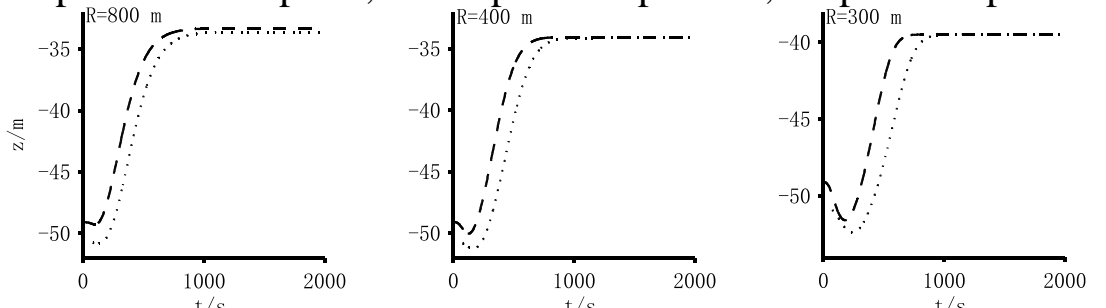

Fig.5. Time history of depth during loop maneuvers for $\mathrm{V}=6 \mathrm{kn}, \mathrm{R}=800,400$, and $300 \mathrm{~m}$. - - - represents point $\mathrm{A}$, : represents point $\mathrm{B}$

As can be seen from Fig. 4, point A (or B) achieves a relatively smaller loop turning radius in the case with a relatively smaller loop maneuver radius of ship. In addition, the loop trajectories of point A get farther and farther from those of point B with reduction in circular maneuver radius of ship.

In Fig. 5, the three set of plots show a similar trend of depth in the time history. During the ship turn, the depth of point $\mathrm{A}$ and $\mathrm{B}$ will increase to a maximum and then rise back to the original straight tow equilibrium depth. Note that the final steady-state depth values of point A and B for R $=300 \mathrm{~m}$ are the same, while those of the other two cases are not. The distinction among the three cases indicates that the array tends to conduct its circular turning motion in a horizontal plane and form a spiral when the ship loop radius reduces to a critical value.

\section{Different current}

Calculations are performed for three ship loop radii $(\mathrm{R}=800,400$, and $300 \mathrm{~m})$ and a fixed tow speed $(\mathrm{V}=18 \mathrm{kn})$ with a steady current that has a magnitude of $1 \mathrm{~m} / \mathrm{s}(+\mathrm{x}$ current or $-\mathrm{x}$ current).
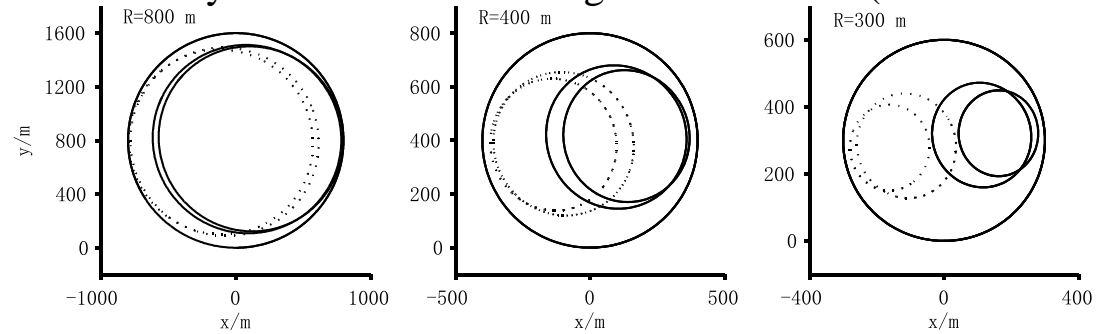

Fig.6. Plan view of loop maneuvers for $V=18 \mathrm{kn}, \mathrm{R}=800,400$, and $300 \mathrm{~m}, \mathrm{c}=-1,1 \mathrm{~m} / \mathrm{s}$. -- represents c $=-1 \mathrm{~m} / \mathrm{s}$, : represents $\mathrm{c}=1 \mathrm{~m} / \mathrm{s}$

Fig. 6 shows an overhead view of the tow ship undergoing steady loop maneuvers in the presence of a current. In this case, there are two simulations with the current in opposite directions (positive x-current and negative x-current). Compared to Fig. 2, evident deviations of point A's and B's trajectories can be seen. No matter the current is positive or negative, the change trend of linear 
array A and B two points is analogue that is offset in the direction of current. As the loop maneuver radius of the tow ship decreases, the area enclosed by the point A's (or B's) turning circle gets smaller, which has similarities with Grosenbaugh's study [7].
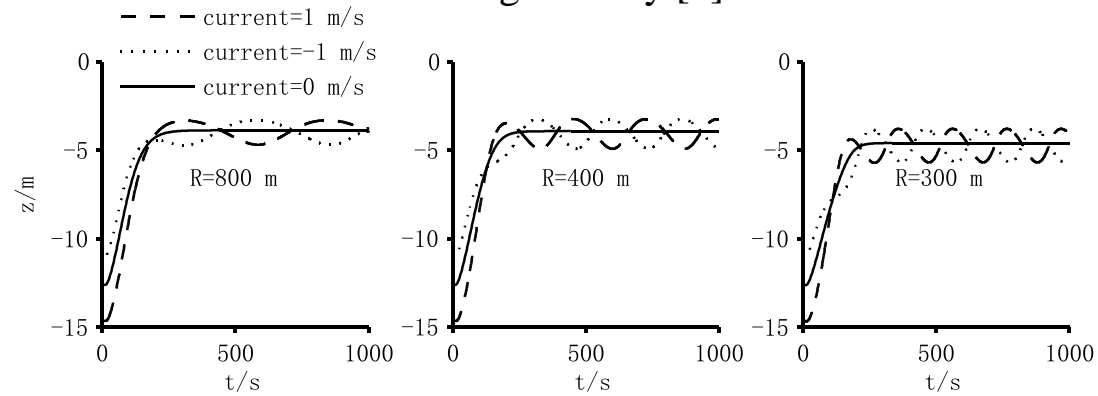

Fig. 7. Time history of point A's depth during loop maneuvers in $\pm \mathrm{x}$-current for $\mathrm{V}=18$ knots, $\mathrm{R}$ $=800,400$, and $300 \mathrm{~m}$. Zero-current results are plotted for reference.

Fig. 7 gives the time history of point $A$ depth during steady-state circular maneuvers in the presence of an x-current. Apart from the change of the starting depth of point A, there are still a few more differences caused by x-current. Once the towed system achieves its steady-state during loop maneuver, the depth of point A oscillates around the zero-current solution, which indicates that the location of the array has a similar variation in the maneuver. Also shown are the phase differences of $180^{\circ}$ between the plots for two opposite direction current. Moreover, as the loop radius of the tow ship decreases, the frequency of the oscillatory motion decreases to a certain extent.
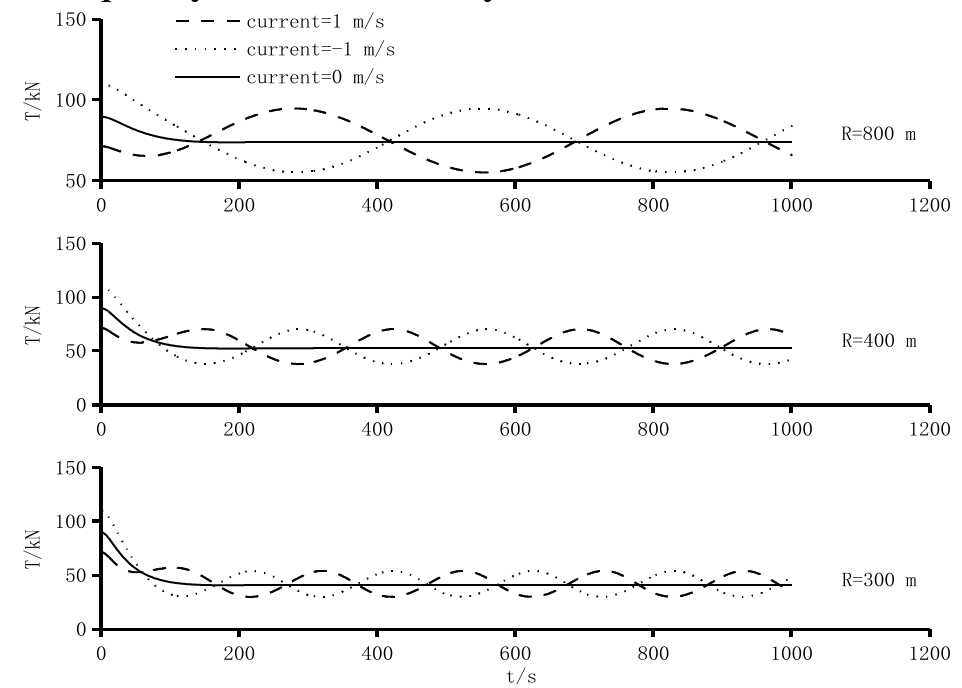

Fig.8. Time history of tow-point tension during steady-state loop maneuvers in $\pm \mathrm{x}$-current for c $= \pm 1 \mathrm{~m} / \mathrm{s}, \mathrm{V}=18$ knots, and selected ship-turning radii.

Fig. 8 shows the time history of tow-point tension during steady-state turning maneuvers for selected ship-turning radii. The differences of tow-point tension caused by $\mathrm{x}$-current are similar to those of point A depth variation shown in Fig. 7. The first obvious effect of $x$-current is to change the initial steady-state tension of tow-point--lower for positive x-current and higher for negative $\mathrm{x}$-current. Once the towed system achieves its steady-state during loop maneuver, the tension value of tow-point oscillates about the zero-current solution. The amplitude of the oscillation becomes smaller with the deduction of the ship-turning radius. Also, a phase difference of $180^{\circ}$ is observed between the cases with two opposite currents.

Considering that the cable-towed acoustic array system starts forming a close-loop with the loop maneuver radius decreasing to a particular value (close-loop radius), The loop critical radius is closely related to line length and towing speed, still the current velocity. No ship-turning radii that used in the above simulations are less than 300 m. Fig. 9 shows the effect of current on close-loop radius. 

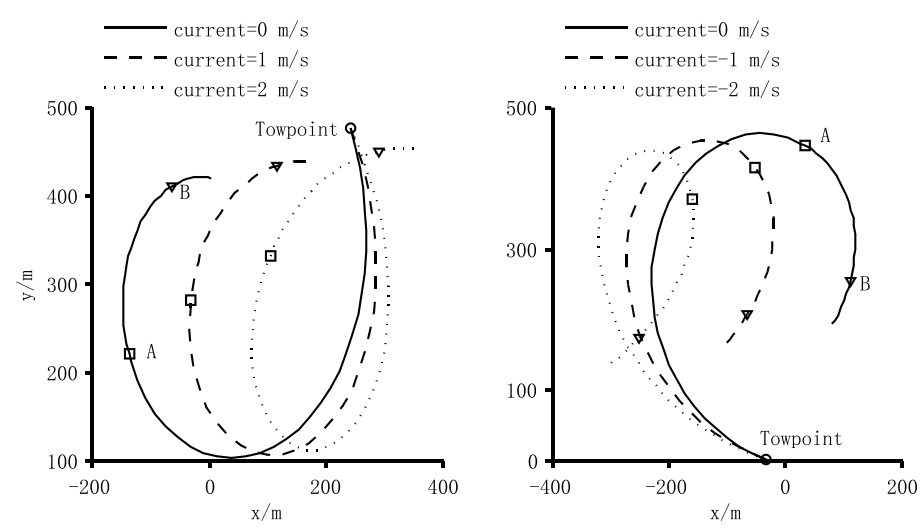

Fig.9. Plan view of cable-towed array in presence of current during constant radius $(R=300 \mathrm{~m})$

It is seen that no close-loop is formed for the towed array in the case of zero-current. For fixed tow speed and ship-turning radius, the towed system starts to form a close-loop for a specific value of current speed. As the current speed increases, the closeness of the system loop is also increased as shown in Fig. 9. The effect of current direction is felt on the time required for the towed system formation the close-loop after the tow ship begins its circular maneuver. On the other hand, current direction has no influence on the magnitude of the critical radius.

\section{Conclusions}

The dynamic response of a cable-towed acoustic array during ship's loop maneuvers is studied and the sensitivity analysis of towing speed, turning radius, and current are discussed in detail. Some general trends are summarized as follows:

(1) The turning radius of the cable-towed acoustic array is insensitive to the variation of towing speed. On the contrary, the depth of the towed array will show a figure-V variation during ship's loop maneuvers with the deduction of towing speed.

(2) As the radius of ship's decreases, that of the towed array's will decrease. Additionally, the trend of the array depth's figure- $V$ variation will also be weaken.

(3) Current is an important factor that affects the towed array's loop maneuver behavior significantly. The towed sonar array will deviate under current. The solution for case with current oscillates around the zero-current solution. The close-loop of the cable-towed acoustic array will decrease with the rise of the current speed.

\section{Acknowledgements}

Funding for this work was provided by the National Natural Science Foundation of China (Grant No. 11272160) and the Youth Project of Natural National Science Foundation of China (Grant No. 51309133).

\section{References}

[1] Ablow C M, Schechter S. Numerical simulation of undersea cable dynamics [J]. Ocean Engineering. 1982, 10(6): 443-457.

[2] Milinazzo F, Wilkie M and Latchman S A. An efficient algorithm for simulating the dynamic of towed cable system [J]. Ocean Engineering, 1987, 14(6): 513-526.

[3] Delmer T N, Stephens T C, Tremills J A. Numerical simulation of cable-towed acoustic arrays [J]. Ocean Engineering. 1988, 15(6): 511-548.

[4] Gobat J I, Grosenbaugh M A. Time-domain numerical simulation of ocean cable structures [J]. Ocean Engineering, 2006, 33(10): 1373-1400.

[5] Choo Y, Casarella M J. Configuration of a towline attached to a vehicle moving in a circular path [J]. Journal of Hydronautics. 1972, 6(1): 51-57. 
[6] Chapman D A. The towed cable behavior during ship turning maneuvers [J]. Ocean Engineering. 1984, 11(4): 327-361.

[7] Grosenbaugh M A. Transient behavior of towed cable systems during ship turning maneuvers [J]. Ocean Engineering. 2007, 37(11-12): 1532-1542.

[8] Kishore S S, Ganapathy C. Analytical investigations on loop-maneuverof underwater towed cable-array system [J]. Applied Ocean Research, 1996, 18: 353-360.

[9] YANG Bing-ka, ZHU Ke-qiang, ZHU Yan-jie, et al. Dynamic response of towed line array [J]. Journal of Hydrodynamics. 2013, 25(4): 616-619. 